ARTICLE

Received 19 Jul 2014 | Accepted 11 Mar 2015 | Published 22 Apr 2015

DOI: $10.1038 /$ ncomms7898

\title{
Regulation of endogenous transmembrane receptors through optogenetic Cry2 clustering
}

\author{
L.J. Bugaj ${ }^{1,2}$, D.P. Spelke ${ }^{1,2}$, C.K. Mesuda ${ }^{3}$, M. Varedi ${ }^{4}$, R.S. Kane ${ }^{5,6}$ \& D.V. Schaffer ${ }^{1,2,3,4,7}$
}

Transmembrane receptors are the predominant conduit through which cells sense and transduce extracellular information into intracellular biochemical signals. Current methods to control and study receptor function, however, suffer from poor resolution in space and time and often employ receptor overexpression, which can introduce experimental artefacts. We report a genetically encoded approach, termed Clustering Indirectly using Cryptochrome 2 (CLICR), for spatiotemporal control over endogenous transmembrane receptor activation, enabled through the optical regulation of target receptor clustering and downstream signalling using noncovalent interactions with engineered Arabidopsis Cryptochrome 2 (Cry2). CLICR offers a modular platform to enable photocontrol of the clustering of diverse transmembrane receptors including fibroblast growth factor receptor (FGFR), platelet-derived growth factor receptor (PDGFR) and integrins in multiple cell types including neural stem cells. Furthermore, light-inducible manipulation of endogenous receptor tyrosine kinase (RTK) activity can modulate cell polarity and establish phototaxis in fibroblasts. The resulting spatiotemporal control over cellular signalling represents a powerful new optogenetic framework for investigating and controlling cell function and fate.

\footnotetext{
${ }^{1}$ Department of Bioengineering, University of California, Berkeley, Berkeley, California 94720, USA. ${ }^{2}$ The UC Berkeley-UCSF Graduate Program in Bioengineering, University of California, Berkeley, Berkeley, California 94720, USA. ${ }^{3}$ Department of Chemical Engineering, University of California, Berkeley, Berkeley, California 94720, USA. ${ }^{4}$ California Institute for Quantitative Biosciences, University of California, Berkeley, Berkeley, California 94720, USA. ${ }^{5}$ Department of Chemical and Biological Engineering, Rensselaer Polytechnic Institute, Troy, New York 12180, USA. ${ }^{6}$ Center for Biotechnology and Interdisciplinary Studies, Rensselaer Polytechnic Institute, Troy, New York 12180, USA. ${ }^{7}$ Helen Wills Neuroscience Institute, University of California, Berkeley, Berkeley, California 94720, USA. Correspondence and requests for materials should be addressed to R.S.K. (email: kaner@rpi.edu) or to D.V.S. (email: schaffer@berkeley.edu).
} 
C ells receive dynamic information via transmembrane receptor signals and integrate these cues to regulate diverse biological processes including cell proliferation, motility, differentiation and death. Currently utilized approaches to study transmembrane receptor signalling ${ }^{1,2}$ often employ overexpression of receptors and receptor fusions, which fundamentally alter how cells respond to extracellular signals and frequently induce hypersensitive or constitutive signal activation $^{3-5}$. Indeed, receptor gene amplification and overexpression is a common driving factor of cancer cell transformation $^{6,7}$. Moreover, current approaches do not provide a high degree of temporal and spatial control that would be desirable to study receptor signalling dynamics and spatially heterogeneous behaviour, respectively.

In recent years, the emerging field of optogenetics has offered several methods to optically control and study numerous signalling phenomena with spatiotemporal precision $^{8-17}$, although each currently relies on the ectopic expression of the signalling protein of interest. In a recent example, we co-opted the inherent blue-light-dependent oligomerization of Arabidopsis Cry2 to regulate clustering and activation of fusion proteins in the cytosol of mammalian cells ${ }^{16}$. In this report, we demonstrate the optogenetic control of endogenous transmembrane receptor activity. Our approach makes use of receptor clustering, which is a natural mechanism of activation for many important transmembrane receptors, including receptor tyrosine kinases, immune receptors (for example, T-cell receptor (TCR), B-cell receptor $(\mathrm{BCR})$ and $\mathrm{Fc}$ receptor $(\mathrm{FcR}))$, Wnt receptors and integrins ${ }^{18-26}$. Specifically, we re-designed and implemented Cry2 clustering to optically target, cluster and regulate endogenous transmembrane proteins in a modular strategy called Clustering Indirectly using Cryptochrome 2, or CLICR.

\section{Results}

CLICR design. In designing CLICR (Fig. 1a), we hypothesized that Cry2 fused to a binding domain (Cry2-BD) possessing limited affinity for a target receptor would remain largely in the cytoplasm in the absence of light. On blue light illumination, clusters of Cry2-BD would assemble and thereby effectively create high local concentrations of the BD that may enable simultaneous binding to multiple receptor endodomains, resulting in receptor clustering and signal activation.

As Cry2 optogenetic clustering had been well characterized using cytoplasmic proteins ${ }^{16}$, we first assessed the feasibility of CLICR by investigating whether we could optically cluster a Cry2-binding target in the cytoplasm and whether indirect clustering could induce signal activation (Fig. 1b, Supplementary Fig. 1). Using a heterodimeric pair of leucine zippers ${ }^{27}$, we grafted one of the LZ helices (LZa) on the Cry2 module fused to mCherry $(\mathrm{mCh})$ and its partner helix $(\mathrm{LZb})$ on a green fluorescent protein (GFP)-LRP6c fusion, whose clustering has been shown to robustly stimulate the $\beta$-catenin pathway signalling ${ }^{16,28}$. A list of fusion architectures examined can be found in Supplementary Table 1, and a complete list of constructs generated for this work can be found in Supplementary Data 1. On co-transfection of the LZ-appended Cry2 and LRP6c fusions into HEK 293T cells, we observed light-induced cluster formation in both the mCherry and GFP channels, and these clusters colocalized, suggesting that Cry2 clusters were successfully clustering GFP-LRP6c fusions via the LZa-LZb interaction (Fig. 1c, Supplementary Fig. 1b).

Cytoplasmic and membrane-targeted signal activation through CLICR. To determine whether the light-stimulated LRP6c clusters were sufficient to induce $\beta$-catenin signalling, we transfected combinations of Cry2- and LRP6c-containing fusion pairs into
$293 \mathrm{~T}$ cells harbouring a $\beta$-catenin-responsive luciferase reporter ${ }^{29}$ (Fig. 1c). Significant increases in $\beta$-catenin signalling in illuminated versus unilluminated samples were observed with all Cry2/LRP6c fusion pairs tested, although this signal was markedly diminished compared with that obtained with a singlechain Cry2-mCh-LRP6c fusion (Supplementary Fig. 1d) ${ }^{16}$, potentially because of LRP6c cluster geometries, expression level differences or differences in efficiencies of two-component versus one-component clustering. We further demonstrated the feasibility and modularity of the CLICR approach by indirectly clustering the Racl GTPase with the same LZ-binding adapters (Supplementary Fig. 1e). Consistent with our previous report of clustering the Rac1 protein ${ }^{16}$, indirect Rac1 clustering induced rapid membrane translocation of the Rac1 (and associated Cry2) fusion (Supplementary Fig. 1f). Together, the LRP6c and Rac1 results demonstrate that CLICR can serve as a modular strategy for clustering binding partners via noncovalent interactions.

We next investigated whether a CLICR-induced avidity increase was sufficient to elicit translocation of a cytoplasmic Cry2 fusion to the plasma membrane, which would be necessary for CLICR activation of membrane receptors (Fig. 1a). We co-transfected cells with a membrane-localized LZb-GFP-CaaX construct, which is lipid-modified at the C-terminal CaaX box, and Cry2-fused to mCherry with the LZa helix at either the C- or $\mathrm{N}$ terminus (Fig. 1d, Supplementary Fig. 2a). In the dark state, LZb-GFP-CaaX appeared membrane-bound, whereas Cry2-LZa fusions were largely cytoplasmic with few regions of visible membrane association, suggesting weak basal affinity of the $\mathrm{LZa}-$ LZb binding partners. On light illumination, both Cry2 fusions exhibited translocation to the cell periphery and to immobilized puncta within the cell body, suggesting strong light-induced binding to cell membranes (Fig. 1e, Supplementary Fig. 2b). To analyse whether CLICR-mediated membrane translocation could induce signalling on binding and clustering of a transmembrane receptor, we replaced the LZb-GFP-CaaX fusion with a GFP$\mathrm{LZb}$ fused to the $\mathrm{C}$ terminus of the full-length LRP6 receptor (Supplementary Fig. 3a), whose clustering induces $\beta$-catenin signalling ${ }^{28}$. LRP6-GFP-LZb was localized to multiple cellular compartments (Supplementary Fig. 3b), and illumination of Cry2 with N- or C-terminally fused LZa (Supplementary Fig. 3c,d), but not with Cry2 lacking the LZa (Supplementary Fig. 3e,f), successfully induced Cry2 translocation and colocalization with LRP6-GFP-LZb as well as activation of the $\beta$-catenin luciferase reporter, although high background signal was observed in the dark (Supplementary Fig. 3g) because of receptor overexpression (Supplementary Fig. 3h). By comparison, a direct LRP6-Cry2 fusion resulted in a constitutive signal that could not be further enhanced by illumination, further representative of artefact that can accompany overexpression of transmembrane receptor constructs (Supplementary Fig. 4). These results both provided support for the CLICR approach and motivated adapting it to activate endogenous receptors and thus overcome overexpression artefact.

Targeting of receptor tyrosine kinases. The receptor tyrosine kinase (RTK) class of receptors is broadly important in regulating proliferation, growth, differentiation, survival and motility, and activation of many RTKs involves lateral interactions including clustering ${ }^{23}$. Furthermore, signalling artefacts are commonly observed on RTK overexpression within cells ${ }^{3,5}$. To target a cell's native RTKs with CLICR, we used the N-terminal srchomology 2 (SH2) domain from PLC- $\gamma$ as our binding domain adapter (Fig. 2a). Since the SH2 domain typically does not bind unphosphorylated peptide sequences, we hypothesized that $\mathrm{SH} 2$ domain clustering may enhance its avidity for unactivated or 
a

CLICR:

Clustering Indirectly using Cryptochrome2

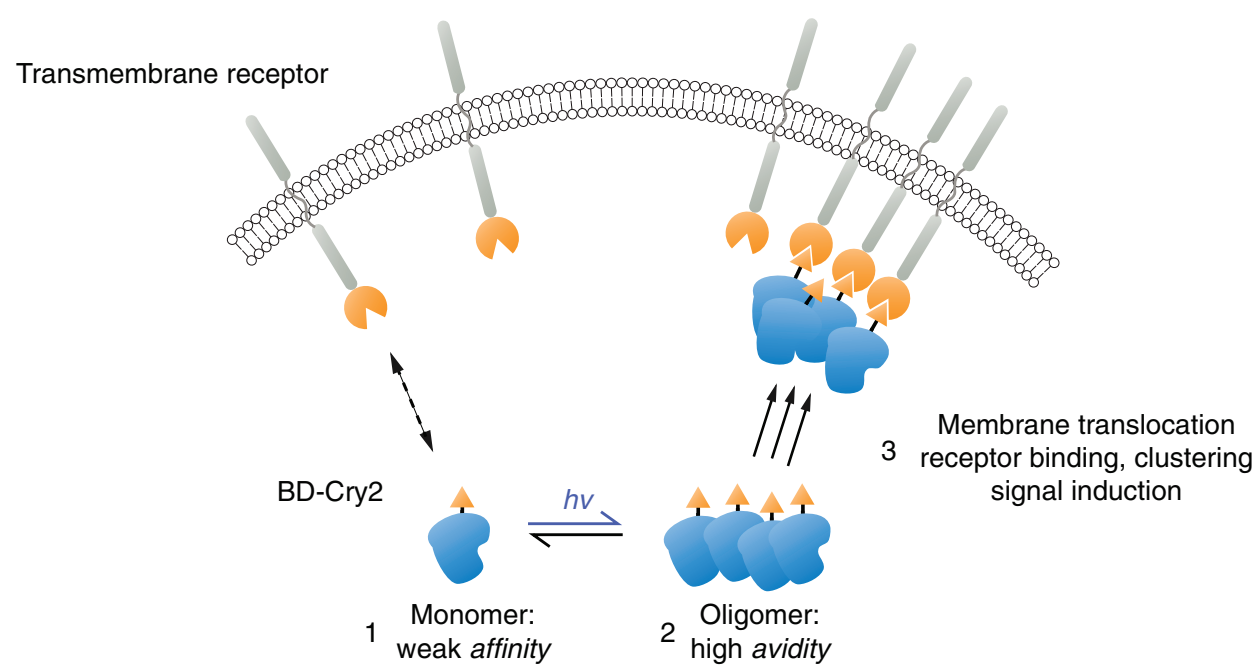

b

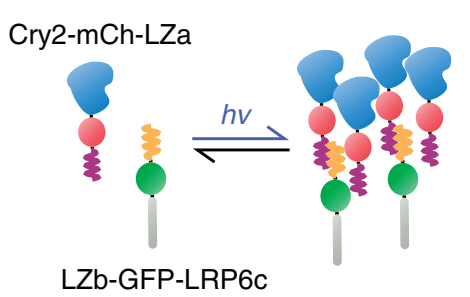

$\begin{array}{cc}\text { Cry2- } & \text { LZb-GFP } \\ \text { mCh-LZa } & \text { LRP6c }\end{array}$

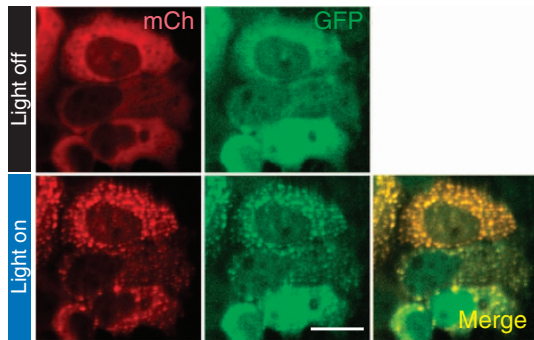

d

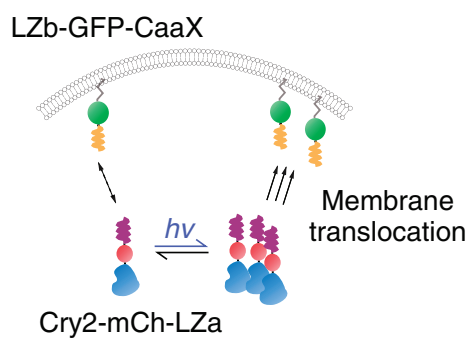

e

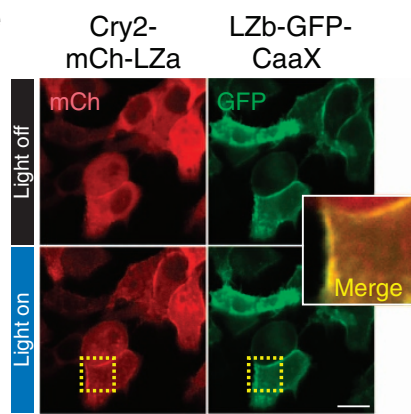

Figure 1 | The CLICR strategy enables Cry2 activation of transmembrane receptors. (a) CLICR allows modular Cry2 clustering and activation of membrane receptors via noncovalent interactions, avoiding complications associated with overexpression of receptor fusions. With CLICR, Cry2 fused to a receptor-targeting binding domain (BD) is expressed in the cytoplasm. In the dark, unclustered state, BD affinity for the receptor is weak and imparts no or little membrane localization. On light-induced clustering, BD-Cry2 oligomers increase local BD concentration, enabling membrane translocation, binding and nucleation of a receptor cluster. (b) CLICR allows clustering of cytoplasmic protein targets. On light activation, the Cry2-mCh-LZa (red) and LZb-GFPLRP6c (green) components co-cluster (c), suggesting successful clustering of noncovalently bound proteins via homo-oligomerization of Cry2. (d,e) CLICR clustering also enables membrane translocation of the initially cytoplasmic Cry2-mCh-LZa. Scale bars, $20 \mu \mathrm{m}$.

weakly activated receptors ${ }^{30}$ and subsequently cluster and activate the RTKs. To test RTK-targeting ability, we expressed mCherryCry2 with an N-terminal SH2 domain $(\mathrm{SH} 2-\mathrm{N})$ in several cell types, including HEK 293Ts, 3T3 fibroblasts and adult neural stem cells (NSCs). In 293Ts, illumination of SH2-N yielded membrane-anchored puncta (Fig. 2b, top row; Supplementary Movie 1). Analogously, 3T3 fibroblasts exhibited rapid localization of SH2-N to membrane-anchored puncta as well as to focal adhesion structures (Fig. 2c, top row, Supplementary Movie 2), and NSCs exhibited light-induced punctate formation in neurite extensions as well as in the cell body, collectively suggesting that $\mathrm{SH} 2-\mathrm{N}$ may target endogenous membrane proteins (Fig. 2d, top row; Supplementary Movie 3). A C-terminal fusion of $\mathrm{SH} 2$ to $\mathrm{Cry} 2-\mathrm{mCh}$ (SH2-C) was also tested and yielded similar phenotypes, although with slower kinetics, and the SH2-N construct was thus used for all further experiments. Treatment with the RTK inhibitor PD-089828 (RTKi) - which inhibits phosphorylation of the RTKs FGFR1, PDGFR $\beta$ and EGFR - markedly reduced SH2-N focal enrichment at the membrane in all three cell types (Fig. $2 \mathrm{~b}-\mathrm{d}$, bottom rows). 
a

Clustered

endogenous RTK

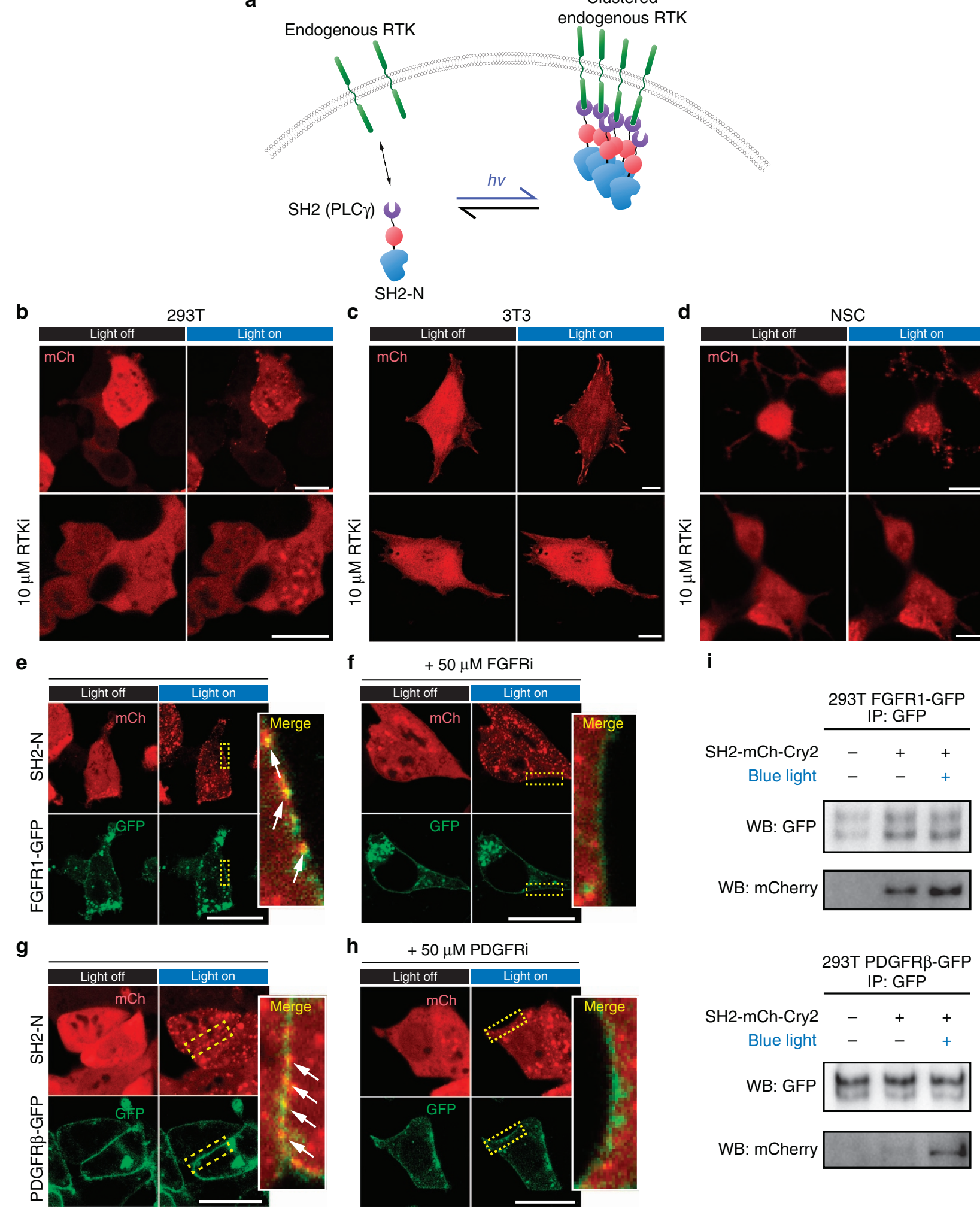

Figure 2 | CLICR can target and cluster endogenous transmembrane receptors. (a) To target endogenous receptors with CLICR, an adapter domain with affinity for native receptors was used in place of the engineered LZa-LZb interaction used previously. The SH2 domain from PLC $\gamma$ was used as a binding domain fused to the $\mathrm{N}$ terminus of mCh-Cry2 (SH2-N) to target endogenous receptor tyrosine kinases. Illuminated SH2-N expressed in 293Ts (b, top panels) localized to membrane-anchored foci. In $3 \mathrm{~T} 3$ fibroblasts (c, top panels), illumination of SH2-N induced translocation to the membrane periphery and to structures resembling focal adhesions. In NSCs (d, top panels), illuminated SH2-N localized to immobilized membrane clusters both in the cell body and in neurites. In all three cell types, inhibition with an FGFR1/PDGFRß/EGFR-specific inhibitor (RTKi) largely abrogated visible SH2-N translocation. (b-d, lower panels). In 293Ts, upon blue light illumination SH2-N colocalized (arrows) to exogenously expressed, full-length FGFR1 (e) and PDGFRß (g) fused to GFP, and this colocalization was abrogated in the presence of inhibitors specific to these receptors (f,h). Scale bars, $20 \mu \mathrm{m}$. Light-stimulated association of SH2-N with either FGFR1-GFP or PDGFR $\beta$-GFP was confirmed through co-immunoprecipitation of SH2-N with the receptors (i). 
These data indicate that strong $\mathrm{SH} 2-\mathrm{N}$ localization is dependent on RTK phosphorylation, and thus SH2-N clusters are indeed targeting endogenous RTK receptors.

To further validate that $\mathrm{SH} 2-\mathrm{N}$ was binding RTKs, we transfected the SH2-N construct into 293Ts stably expressing GFP-tagged, full-length FGFR1 or PDGFR $\beta$ receptors, representing two RTKs that bind the PLC $\gamma \mathrm{SH} 2$ domain ${ }^{31,32}$. In cells expressing ectopic FGFR1-GFP, the SH2-N construct exhibited light-dependent membrane translocation and formation of puncta that colocalized with GFP (Fig. 2e). Addition of $50 \mu \mathrm{M}$ FGFR1-4 inhibitor (PD-166866, FGFRi) abrogated this colocalization (Fig. 2f). Similar behaviour was obtained in cells expressing PDGFR $\beta$-GFP, where light-induced SH2-N colocalization with PDGFR $\beta$-GFP (Fig. 2g) was abrogated in the presence of $50 \mu \mathrm{M}$ PDGFR- $\alpha$ and $-\beta$ inhibitor (CAS 205254-94-0, PDGFRi; Fig. 2h). Light-promoted SH2-N binding to FGFR1 and PDGFR $\beta$ was further established through co-immunoprecipitation of SH2-N with either the FGFR1 or PDGFR $\beta$ receptors (Fig. 2i). Together, these results indicate that the cytoplasmic SH2-N construct can successfully target native transmembrane RTKs via CLICR in response to light.

Optogenetic activation of endogenous transmembrane receptors. We next investigated whether SH2-based CLICR targeting of endogenous RTKs is capable of signal activation. Illumination of $3 \mathrm{~T} 3$ fibroblasts expressing $\mathrm{SH} 2-\mathrm{N}$ yielded a broad increase in tyrosine phosphorylation, compared with unilluminated and RTKiinhibited controls (Fig. 3a). Furthermore, analysis of canonical RTK signalling pathways showed an increase in levels of phospho-Akt and phospho-Erk1/2 after $15 \mathrm{~min}$ of blue light illumination, a difference abrogated in the presence of the RTKi inhibitor. A central benefit of employing CLICR for RTK activation is the lack of receptor overexpression, which may introduce artefacts including hypersensitity to extracellular ligand. Indeed, in contrast to cells overexpressing a PDGFR $\beta$-Cry2 fusion, $\mathrm{SH} 2-\mathrm{N}$-expressing cells retained native sensitivity to extracellular PDGF-BB (Supplementary Fig. 6).

We further investigated whether CLICR clustering of the PLC $\gamma$ $\mathrm{SH} 2$ domain demonstrated selectivity towards individual receptors. Since activated PDGFR $\beta$ has been shown to colocalize with focal adhesions through interaction with focal adhesion kinase ${ }^{33}$, we hypothesized that endogenous PDGFR $\beta$ activation was largely responsible for the CLICR-induced signal activation and focal adhesion translocation of SH2-N in our study (Fig. 2c). To address this possibility, we examined the light-induced translocation of $\mathrm{SH} 2-\mathrm{N}$ in the presence of pathway-specific inhibitors. In 3T3 fibroblasts, SH2-N exhibited a rapid, light- induced localization to the plasma membrane and focal adhesion structures. Moreover, the 10- $\mu \mathrm{M}$ PDGFRi treatment largely inhibited focal adhesion translocation of $\mathrm{SH} 2-\mathrm{N}$ (Supplementary Fig. 5), whereas 10- $\mu \mathrm{M}$ FGFRi had minimal observed effect on focal $\mathrm{SH} 2-\mathrm{N}$ localization, suggesting that translocation was indeed PDGFR $\beta$-dependent and that CLICR clustering was modulating PDGFR $\beta$ activity.

In general, activated PDGFR colocalization with FAK within focal adhesions has been shown to modulate FAK activation, focal adhesion turnover and cell migration ${ }^{34}$. In addition, PDGFR $\beta$-induced membrane recruitment of phosphatidylinositol3-kinase (PI3K) leads to conversion of phosphatidylinositol-4,5bisphosphate (PIP2) to phosphatidylinositol-3,4,5-trisphosphate $(\mathrm{PIP} 3)^{35}$, whose localization at the leading edge of cells is a key factor in cellular polarity establishment and PDGFR-dependent fibroblast migration. We found that in fibroblasts, SH2-N translocation was accompanied by filopodial and lamellipodial extension, often followed by cellular polarization and movement (Fig. 3b, Supplementary Movie 4). This polarization was dependent on PI3K activity, since addition of the PI3K inhibitor wortmannin led to only transient lamellipodial extensions (Supplementary Fig. 6a, Supplementary Movie 5), which then rapidly stopped protruding or collapsed. PI3K activity was thus required to sustain membrane extensions and establish morphological polarity, consistent with CLICR-mediated activation of PDGFR $\beta$ and PI3K.

We next quantified these PDGFR- and PI3K-dependent effects. Lamellipodial extension induced by whole-field illumination was observed in 78\% of cells expressing SH2-N, and RTKi inhibition significantly reduced this response to $17 \%$ of cells (Fig. $3 \mathrm{c}$, with significance determined through nonoverlapping $95 \%$ confidence intervals). Likewise, PDGFRi significantly decreased the number of cells extending light-induced lamellipodia from 78 to $37 \%$. In contrast, FGFRi had no effect on cell morphological responses. In cells that did exhibit lamellipodial response, we quantified the time interval from the start of blue light exposure to the first apparent lamellipodial extension. Untreated cells extended initial lamellipodia with an average interval of $123 \mathrm{~s}$, while RTKi or PDGFRi significantly delayed such lamellipodial extension in responding cells to 481 and 300 s, respectively (Fig. 3d, analysis of variance (ANOVA) with Tukey post hoc analysis, $P<0.0001$ ). In contrast, FGFRi treatment had no apparent effect on lamellipodial extension timing. We then examined the fraction of cells that established polarity and initiated migration on light treatment, as observed by inspecting lamellipodial extension coupled with trailing edge retraction and morphological reorientation. Sixtyfive percent of uninhibited fibroblasts exhibited polarity

Figure 3 | CLICR allows photoactivation of endogenous RTKs in fibroblasts and enables PDGFRß-dependent phototaxis. Western blot analysis (a) of serum-starved fibroblasts treated for $10 \mathrm{~min}$ with blue light shows broad upregulation of tyrosine phosphorylation in illuminated versus unilluminated samples, and this difference is abrogated by $10 \mu \mathrm{M}$ RTKi treatment. Samples grown in $2 \%$ serum were used as a positive control. In particular, light-treated samples demonstrated an RTK-dependent increase in Akt and Erk1/2 phosphorylation. (b) Time-lapse imaging of SH2-N-expressing fibroblasts shows lamellipodial extension and cell repolarization upon whole-field blue light exposure. (c) The proportion of cells extending lamellipodia in response to wholefield illumination was reduced by broad RTK or PDGFR inhibition, but not FGFR inhibition. Error bars represent $95 \%$ confidence intervals, $n=24-83$ cells per condition. (d) Among cells extending light-induced lamellipodia under broad RTK inhibition or PDGFR inhibition, a longer delay before initial lamellipodial extension was evident, while FGFR inhibition did not induce a delay in extension. Graph shows means \pm 1 s.d., $n=4-58$ cells per condition. ${ }^{\star \star \star} P<0.001$ by one-way ANOVA with Tukey pairwise analysis. (e) The proportion of cells exhibiting light-induced polarization was reduced by the RTKi or PDGFRi treatment, and slightly reduced by FGFRi treatment. Error bars represent $95 \%$ confidence intervals, $n=24-83$ cells per condition. (f) Western blot analysis shows that illuminated SH2-N expressing cells exhibit Akt activation under mock and FGFRi treatment. PDGFRi and RTKi treatment abrogate Akt activation. (g) Focal illumination enabled the spatial definition of the lamellipodial extension region coupled with re-establishment of a PIP3 gradient at the site of illumination (arrows), as observed through a PH(Akt)-Venus biosensor. (h) CLICR-enabled focal activation of lamellipodial extension and polarity establishment allows the effective rewiring of PDGFR-dependent chemotaxis to respond to light. Fibroblasts co-expressing SH2-N and Lifeact-Venus were illuminated focally, and actin polymerization, lamellipodial extension and cell motility were induced in the direction of the illuminated region. Illuminating the trailing edge-induced re-polarization and a reversal of migration direction. Scale bars, $20 \mu \mathrm{m}$. To enhance visualization, excitation laser intensity was increased in bottom panels to compensate for photobleaching. 
establishment, and this response was significantly suppressed to 17 and $12 \%$ of cells treated with RTKi and PDGFRi, respectively (Fig. 3e, significance determined through nonoverlapping 95\% confidence intervals). By comparison, FGFRi treatment only moderately reduced light-dependent polarity induction $(47 \%$ of cells). These results were further corroborated through western blotting, as light-induced Akt activity was observed in uninhibited and FGFRi-inhibited cells, but was absent in RTKi- and PDGFRiinhibited cells (Fig. 3f). Collectively, although not demonstrating unique specificity of receptor targeting, these data demonstrate the ability of $\mathrm{SH} 2-\mathrm{N}$ to modulate endogenous PDGFR $\beta$ activation within $3 \mathrm{~T} 3$ fibroblasts.
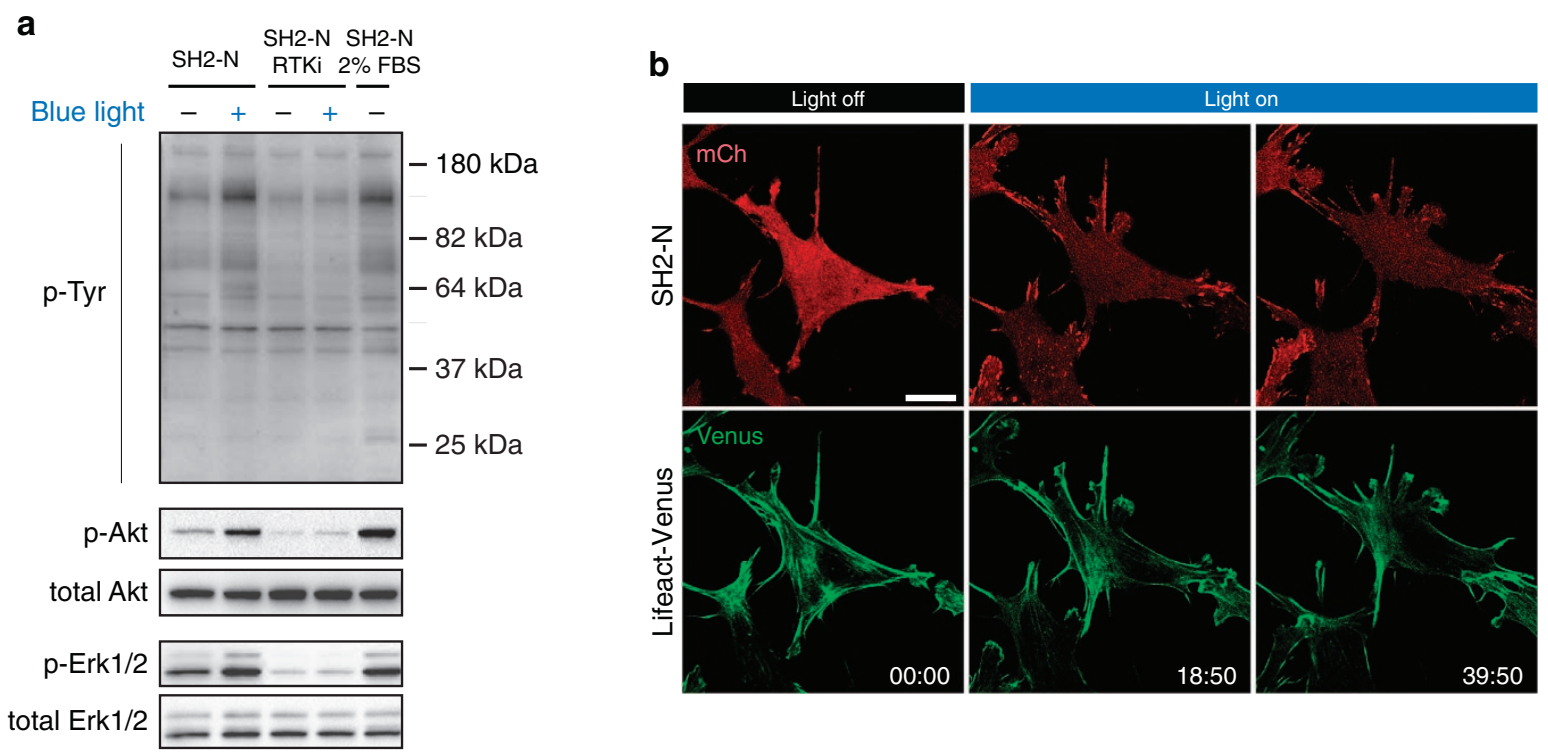

C
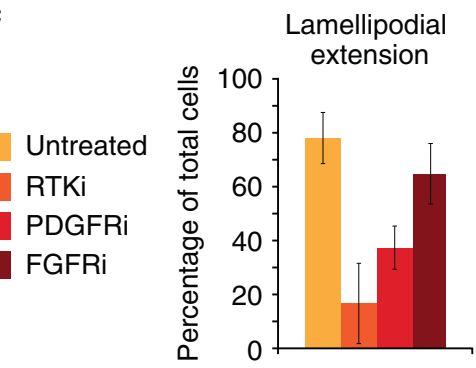

d

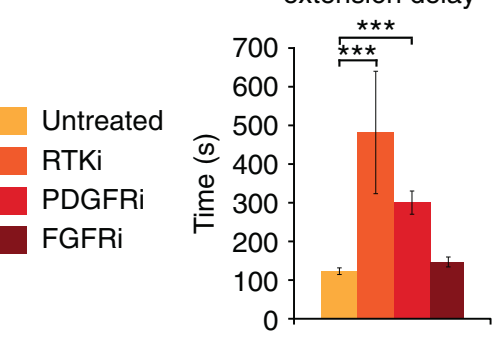

e

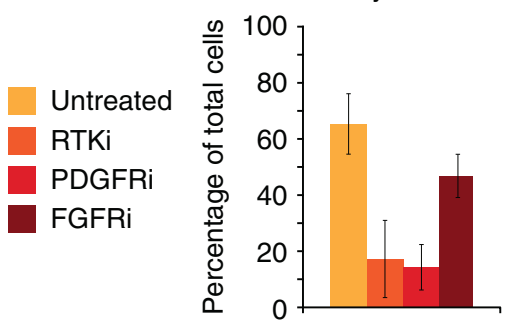

\section{f}

SH2-N SH2-N SH2-N SH2-N PDGFRi FGFRi RTKi

Blue light $\frac{{ }_{-}++}{-+} \overline{-+}$

$$
\text { p-Akt }
$$

Total Akt

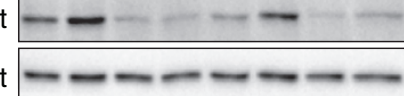

g
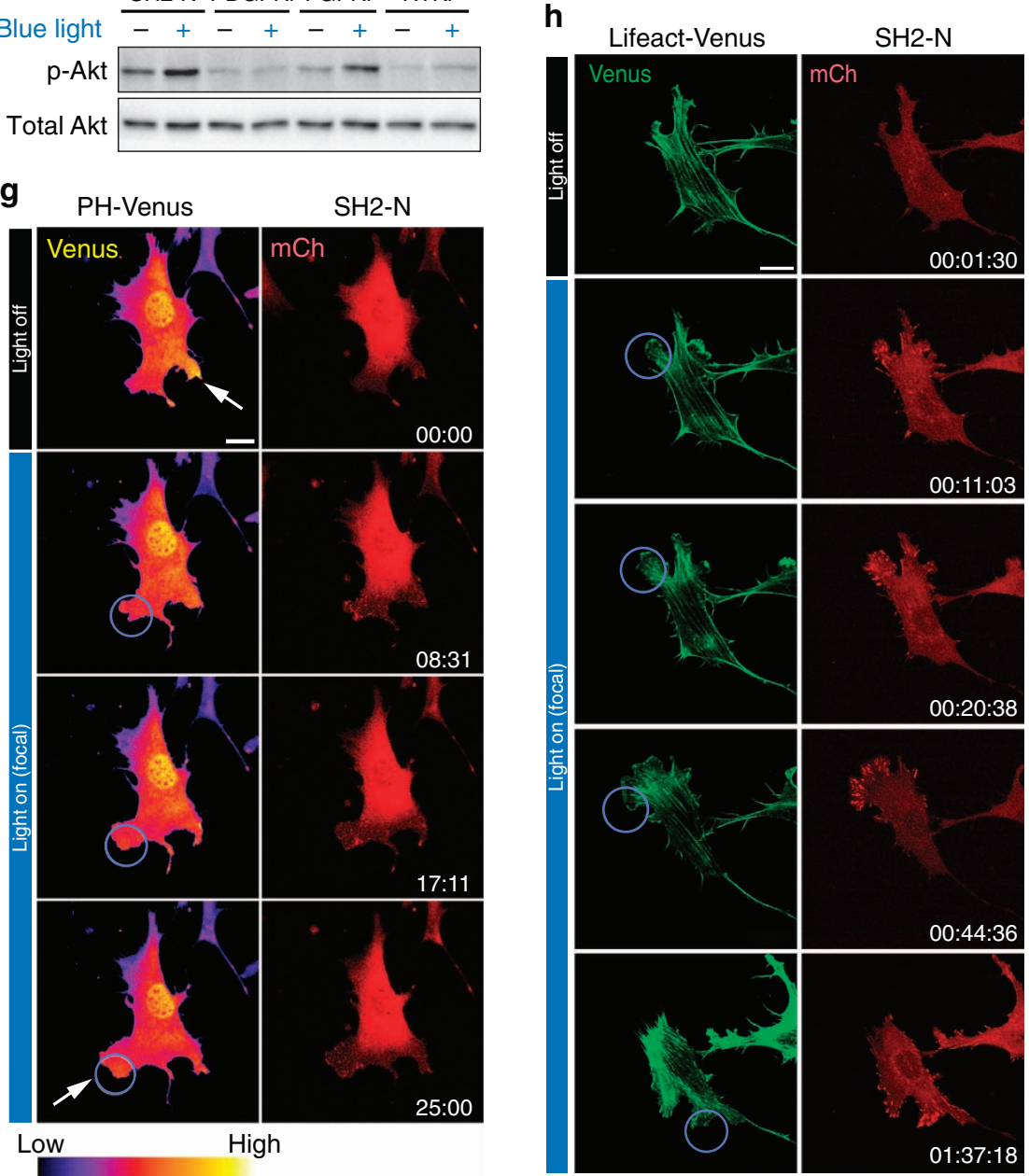
Since PDGFR $\beta$ is a key mediator of fibroblast chemotaxis ${ }^{36}$, we next sought to exploit the spatiotemporal precision of CLICR and rewire a cell's chemotactic circuitry to regulate migration in response to a light signal instead of a chemical one. We hypothesized that focal blue light illumination would establish and define a local PIP3 gradient, and that this would allow directed polarization, actin polymerization and motility of the cell. In fibroblasts co-expressing $\mathrm{SH} 2-\mathrm{N}$ and $\mathrm{PH}-$ Venus, which comprises Venus fused to the pleckstrin homology $(\mathrm{PH})$ domain of Akt and is an established biosensor of PIP3 production ${ }^{37}$, the $\mathrm{PH}-\mathrm{Venus}$ was initially inhomogeneously distributed, indicating initial polarization of PIP3 within the cell. Illuminating the cell in a region not exhibiting initially high levels of PIP3-induced membrane ruffling and lamellipodial extension towards the illumination region, coupled with a redistribution of $\mathrm{PH}-\mathrm{Venus}$ (Fig. 3g, Supplementary Movie 6). In particular, the extending lamellipodium exhibited high levels of PH-Venus recruitment, and within minutes the cell began moving towards the light, establishing a PIP3 gradient and a new leading edge as defined by the illumination region (Fig. 3g, Supplementary Movie 6). In the presence of the PI3K inhibitor wortmannin, however, focal illumination was unable to induce the sustained extension of the illuminated region and establishment of a PIP3 gradient, confirming that local PIP3 production, polarization and motility were PI3K-dependent (Supplementary Fig. 6b, Supplementary Movie 7). In addition, focal blue light illumination of SH2-N cells co-expressing a Lifeact-Venus probe for F-actin stimulated initial lamellipodial actin polymerization, membrane ruffling and extension within $30 \mathrm{~s}$ along with a coordinated initiation of trailing edge retraction (Fig. 3h, Supplementary Movie 8). The cell proceeded to phototax in the direction of the initial illumination region until the light stimulus was reoriented, at which point the established lamellipodium collapsed, and the cells repolarized to migrate in the newly defined direction (Fig. 3h, Supplementary Movie 8).

CLICR can target diverse transmembrane receptors. Finally, to demonstrate that CLICR may be a highly modular method for regulating diverse endogenous pathways, we devised a method to target $\beta$-integrin receptors, which participate in cellular adhesion complexes in their active state and can diffuse freely in the membrane in an inactive state. The important role of clustering in integrin affinity and activation ${ }^{38-40}$ renders endogenous integrins attractive CLICR targets. For the binding adapter, we used the phosphotyrosine-binding-like F3 domain from talin, which binds $\beta 3$-integrin cytoplasmic tails ${ }^{41}$ and whose expression in a Cry2 fusion yielded no observed impact on basal cell signalling (Supplementary Fig. 8). Co-immunoprecipitation of F3-mChCry2 (F3-N) fusion with a co-expressed integrin- $\beta 3-\mathrm{GFP}$ fusion revealed a strong light-dependent association, suggesting that F3 may be used as a binding adapter for targeting integrin- $\beta 3$ clustering (Fig. 4a). Illumination of F3-N in fibroblasts yielded rapid punctate formation and notable motion of the induced puncta along defined intracellular trajectories (Fig. 4b, Supplementary Movie 9). These phenotypic results were distinct from those described for $\mathrm{SH} 2-\mathrm{N}$ and were absent in cells expressing mCherry-Cry2 without the F3 domain (Fig. 4b). 3T3 cells co-expressing both $\mathrm{F} 3-\mathrm{N}$ and integrin- $\beta 3$ revealed strong co-clustering of the two constructs under light illumination, with a reversion to diffuse localization in the dark (Fig. 4c). This co-clustering was observed in all 116 co-expressing cells in which the F3-N construct exhibited cluster formation (Fig. 4d). Furthermore, analysis of over 400 clusters within cytoplasmic regions of these cells revealed that every F3-N cluster colocalized with an integrin- $\beta 3$ cluster (Fig. 4e,f), suggesting a strong ability for the F3 domain to enable integrin- $\beta 3$ clustering via CLICR and overall demonstrating the generality of the CLICR approach for targeting diverse membrane targets ${ }^{42}$.

\section{Discussion}

Spatially defined and rapidly time-varying signals are the key regulatory factors in diverse biological processes including cell migration $^{43}$, asymmetric cell division ${ }^{44}$, differentiation ${ }^{5}$ and apoptosis ${ }^{45}$. The difficulties of perturbing biological systems with such spatiotemporal precision, however, often preclude our understanding of how dynamic signals regulate cellular function. Optogenetic tools address this need. Although methods for optical protein homodimerization ${ }^{15,46}$, heterodimerization ${ }^{11-14}$ and homo-oligomerization ${ }^{16}$ of ectopically expressed proteins have been developed, ectopic expression of many signalling proteins-particularly transmembrane receptors-can fundamentally alter how the system of study behaves, potentially confounding conclusions reached with even the most sophisticated perturbative techniques. We devised the CLICR method to address this dilemma.

The CLICR strategy provides a modular, single construct system to optically cluster and activate transmembrane receptors from the cytosol and is a powerful method for reversibly modulating endogenous transmembrane receptor activity. We accomplished this by pairing Cry2 clustering with a binding adapter for our target receptors. Although we envisioned the ideal CLICR adapter to have weak target affinity in the monomeric state and strong avidity on clustering, it is possible that the CLICR strategy would also work for adapters with strong target affinities. In this case, Cry2-BD would bind the target in the monomeric dark state, and light would induce clustering of these Cry2-BD:target complexes. Indeed, this would be analogous to activation of direct Cry2-receptor fusions $s^{47,48}$ with the added benefit of targeting endogenous pools of signalling proteins. We note that we do anticipate some levels of basal (lightindependent) interaction of our LZa-LZb-binding adapters in the dark state in Fig. 1 and Supplementary Figs 1-3. Our ability to enhance these interactions and observe light-dependent phenotypes further suggests that CLICR can work for binding adapters with a range of binding affinities.

We present CLICR here as a proof of concept strategy demonstrating the feasibility of targeting and activating endogenous receptor activity with light. Since the SH2-N and F3-N constructs bind endogenous phosphotyrosine sites and can therefore have an impact on and be subjected to the native regulation of those phosphorylation sites, we stress that the orthogonality and appropriateness of these and similar tools must be empirically validated for each system before use. Development of alternative, 'orthogonal' binding domains, for example an intracellular antibody domain specifically evolved to target a receptor endodomain ${ }^{49}$, may in the future offer additional versatility, specificity and reduction of basal signal perturbation. Studies should also include controls for Cry2 expression. Although it is unclear to what extent this plant protein domain interacts with mammalian cell machinery, at least one interaction has already been identified ${ }^{50}$.

While our report was under revision, several studies that also further developed optogenetic Cry2 clustering were published. One group applied Cry2 homo-oligomerization for optogenetic activation of overexpressed receptors 47,48 . The demonstrated ability to apply this strategy to multiple RTKs makes this a powerful approach, although we again caution that receptor overexpression often leads to enhanced signalling artefacts (Supplementary Figs $3 \mathrm{~h}, \mathrm{~g}, 4$ and 6 ), potentially altering the system of study. An additional study engineered Cry2 
a

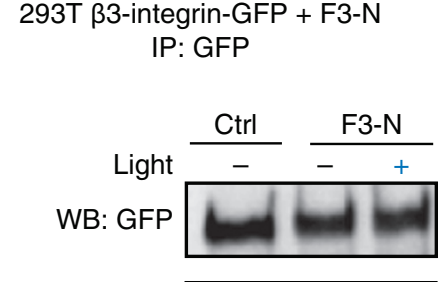

WB: mCherry

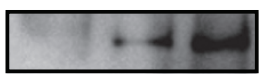

b

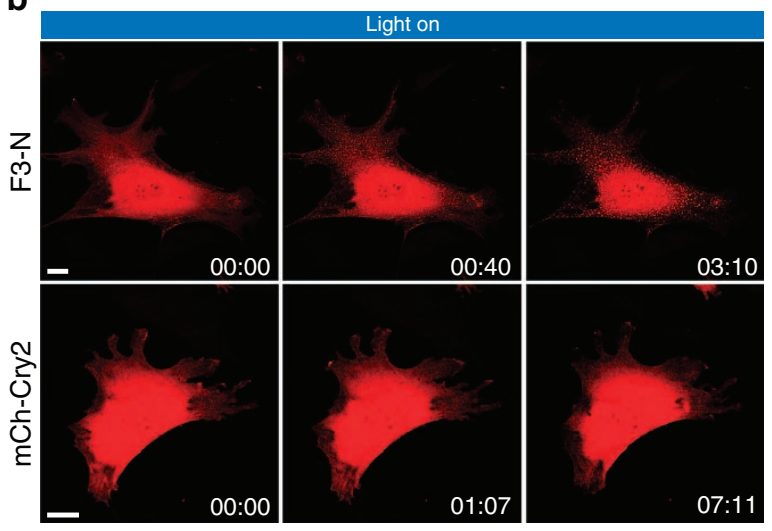

c
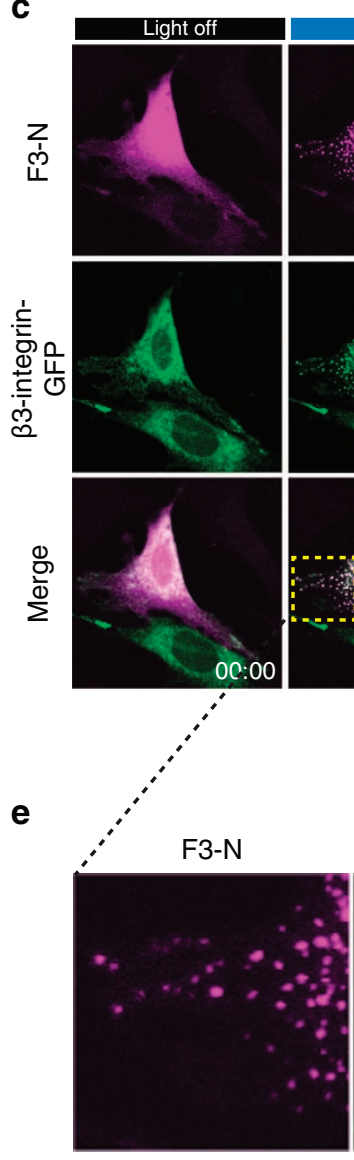
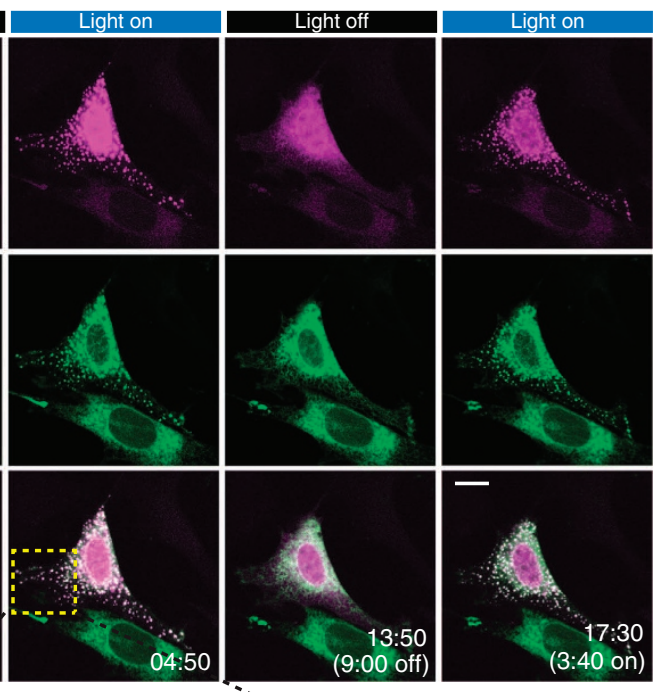

$\beta 3$-integrinGFP

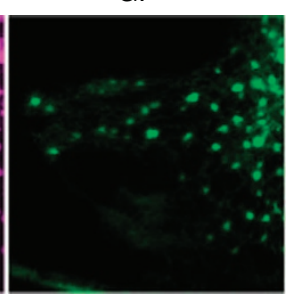

d
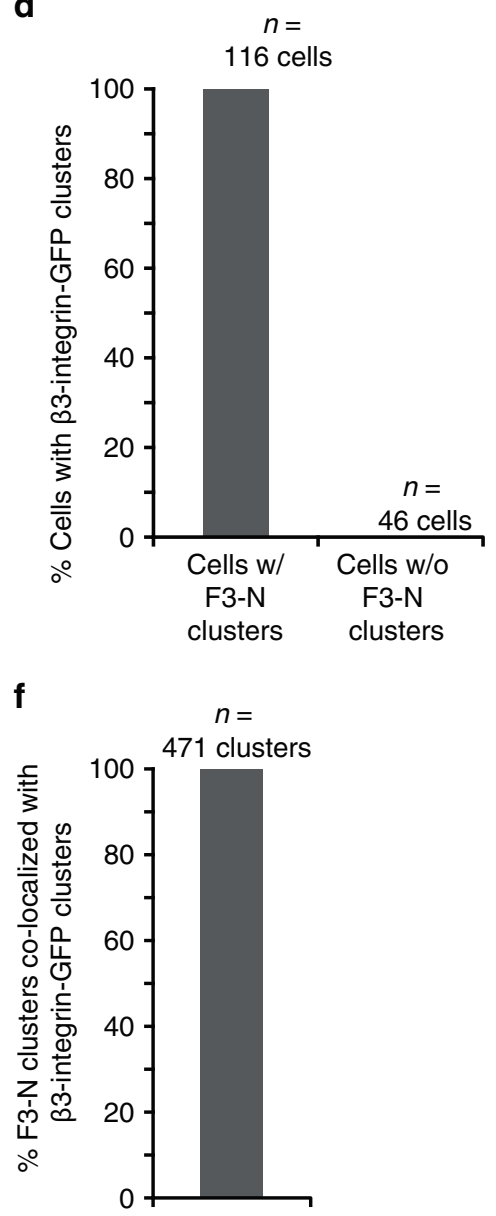

Figure 4 | CLICR targeting and clustering of $\beta$-integrins. (a) Co-immunoprecipitation of the talin F3 domain fused to mCh-Cry2 (F3-N) co-expressed with integrin- $\beta 3$-GFP in HEK 293 T cells reveals strong association of the constructs under blue light exposure. (b) Fibroblasts expressing F3-N show lightinduced formation of puncta that move within the cell along defined linear trajectories (see Supplementary Movie 9). (c) Co-expression of F3-N and integrin- $\beta 3$-GFP in 3 T3 cells demonstrates a high degree of co-clustering of the two constructs under blue light, with reversion of clusters to a diffuse state in the dark. (d) All co-expressing cells in c that displayed F3-N clustering exhibited concomitant $\beta 3$-integrin co-clustering. Further analysis of quantifiable cellular regions (e) revealed that all observable F3-N clusters co-clustered with integrin- $\beta 3$-GFP (f). Scale bars, $20 \mu \mathrm{m}$.

(Cry2-olig) ${ }^{51}$ to form larger clusters than reported with wild-type Cry2 (ref. 16) and used this mutant to sequester and interfere with the function of endogenous proteins. This expansion of Cry2-clustering variants presents a choice for researchers based on their optogenetic needs. For studies seeking to sequester and inhibit protein function, large clusters with slow OFF-kinetics, such as those provided by Cry2-olig, may be ideal. For studies requiring temporally precise activation of endogenous proteins with low background activation, the weaker-associating wild-type Cry2 with faster OFF faster kinetics may be more appropriate.

In sum, receptor overexpression can often elevate pathway signalling above the physiologically basal state and/or alter signal response dynamics. As an alternative, our demonstrated activity, modularity and versatility of CLICR across numerous cell types suggests its utility as an optogenetic tool to manipulate endogenous protein activity across biological systems. 


\section{Methods}

DNA vector assembly. All vectors were constructed using the CPEC method ${ }^{52}$ and through standard restriction enzyme cloning ${ }^{16}$. Full sequences and descriptions of all constructs used are provided in Supplementary Data 1. The LZa and LZb heterodimeric leucine zipper pair corresponds to leucine zipper pair (vii), as described ${ }^{27}$, and was synthesized through primer overlap extension. We received the full-length LRP6 construct from Dr Xi He, the Racl construct from Dr G. Steven Martin, the Akt construct from Dr S. Ferguson, the PDGFR $\beta$, and 7x TCF constructs from Addgene (no. 23893 and no. 24308, respectively), and the $P L C \gamma-1$ construct from the DNASU plasmid repository (clone IDs HsCD00321979 and HsCD00435250, respectively). The FGFR1 construct was cloned from a rat NSC cDNA library.

Cell culture, reagents, plasmid transfection and viral transduction. HEK 293Ts were cultured in Dulbecco's Modified Eagle Medium (DMEM, Corning Cellgro) with $10 \%$ fetal bovine serum (FBS; Life Technologies) and $1 \%$ penicillin/streptomycin (P/S; Life Technologies) at $37^{\circ} \mathrm{C}$ and $5 \% \mathrm{CO}_{2}$. NIH $3 \mathrm{~T} 3$ fibroblasts were cultured in DMEM supplemented with $10 \%$ bovine calf serum and $1 \% \mathrm{P} / \mathrm{S}$. Rat hippocampal adult NSCs ${ }^{53}$ were cultured on polystyrene plates coated with polyornithine and $5 \mu \mathrm{g} / \mathrm{ml}$ laminin (Life Technologies), with DMEM/F12 (1:1) highglucose medium containing N-2 supplement (both from Life Technologies) and $20 \mathrm{ng} / \mathrm{ml}$ recombinant human FGF-2 (Peprotech). Transient plasmid transfection was performed using the calcium phosphate method. Retroviral and lentiviral packaging and transduction were performed as described ${ }^{54}$. Briefly, VSVGpseudotyped virus was packaged in $293 \mathrm{~T}$ cells. Cell culture medium from virusproducing cells was collected on day 2 and day 3 post transfection, sterile-filtered, and purified through ultracentrifugation and resuspension in PBS. All retroviral constructs were based on the CLPIT retroviral vector and were packaged in the presence of $3 \mu \mathrm{g} / \mathrm{ml}$ doxycycline to repress transgene expression in order to maximize viral titre. RTKi (PD-089828), PDGFRi (CAS 205254-94-0, IC $_{50}=50 \mathrm{nM}$ for PDGFR- $\beta$ ) and FGFRi (PD-166866, $\mathrm{IC}_{50}=60 \mathrm{nM}$ for FGFR1) were obtained from Millipore.

Luciferase assay. HEK $293 \mathrm{~T}$ cells stably transduced with a $7 \times \mathrm{Tcf}$ luciferase reporter were seeded in black-walled 96-well plates. When $\sim 50 \%$ confluent, the cells were transfected with $5 \mathrm{ng}$ of each expression vector diluted in $150 \mathrm{ng}$ pBluescript. Cells were given $24 \mathrm{~h}$ to express protein and were then illuminated overnight for $16 \mathrm{~h}$ with one 500-ms pulse of light-emitting diode (LED) light every $10 \mathrm{~s}$ as administered by a custom LED illumination device ${ }^{16}$. After illumination, cells were lysed, and luciferase was measured with the Luc-screen Firefly Luciferase Gene Reporter System (Life Technologies).

Confocal microscopy. Time-lapse microscopy of activated Cry2 fusions was performed on a Zeiss LSM 710 AxioObserver confocal microscope with full incubation chamber in conjunction with the Zeiss ZEN software. Cry 2 translocation experiments were carried out at $25^{\circ} \mathrm{C}$, while experiments examining cell motility and polarity were carried out at $37^{\circ} \mathrm{C}$ and in $5 \% \mathrm{CO}_{2}$. EGFP, Venus and mCherry were visualized with 488,514 and $561 \mathrm{~nm}$ laser excitation, respectively, through either a $\times 40$ or $\times 63$ oil immersion objective. Whole-field Cry 2 activation was achieved using 450- or 488-nm illumination. Spatially defined activation was carried out under whole-cell imaging of Venus with a 514-nm laser in conjuction with focal illumination using the FRAP application with 458- or 488-nm laser light at $1-5 \%$ power and $10 \mu$ s dwell time per pixel in a $10-\mu \mathrm{m}$ diameter region. For whole-field observation of lamellipodial induction and polarity establishment, cells were excited every $13 \mathrm{~s}$ with 488 -nm light, and mCherry was imaged simultaneously.

Image analysis. For whole-field observation of lamellipodial induction and polarity establishment, cells were simultaneously excited with 488-nm light and imaged for mCherry and Lifeact-Venus localization. Time-lapse image stacks were assembled in ImageJ, and each cell was examined for the appearance of new lamellipodia and for the time elapsed from the start of blue light excitation to initial lamellipodial appearance. To assess polarity establishment, cells were scored for the presence of lamellipodial formation coupled with visible trailing edge retraction and morphological reorientation.

Western blotting. 3T3s expressing $\mathrm{SH} 2-\mathrm{N}$ were seeded in $35-\mathrm{mm}$ plates at $50 \%$ confluence and $2 \%$ FBS. After $24 \mathrm{~h}$, the medium was exchanged, and cells were serum-starved for $16 \mathrm{~h}$. Positive control cells remained in $2 \%$ serum. RTKi $(10 \mu \mathrm{M})$ was added $30 \mathrm{~min}$ before illumination. Light-treated plates were illuminated with a 19 LED array (ledlight.com, cat. no. 28345) at $37^{\circ} \mathrm{C}$ in a standard cell culture incubator with a $500-\mathrm{ms}$ pulse every $2 \mathrm{~s}$ for $15 \mathrm{~min}$. Cells were immediately lysed in cold RIPA buffer containing protease and phosphatase inhibitors, and were separated electrophoretically on a 10\% SDS-PAGE gel. Proteins were then blotted on a nitrocellulose membrane and probed for phospho-Tyrosine (P-Tyr-1000, Cell Signaling no. 9411, 1:2,000 dilution), phospho-Akt (Cell Signaling no. 4060, 1:2,000 dilution), total Akt (Cell Signaling no. 9272, 1:1,000 dilution), phospho-Erk1/2 (Cell Signaling no. 4370, 1:2,000 dilution) and total Erk1/2 (Cell Signaling no. 4695,
1:1,000 dilution). For experiments examining signalling artefact, cells were seeded in $10 \%$ serum and starved overnight. Cells were then collected or stimulated with varying concentrations of PDGF-BB for $15 \mathrm{~min}$ before harvesting and performing SDS-PAGE and western blot. Phospho-serine (Abcam no. ab9332, 1:100 dilution), phospho-thereonine (Cell Signaling no. 9381, 1:1,000 dilution) and phospho-tyrosine (P-Tyr-1000, Cell Signaling no. 9411, 1:2,000 dilution) were used to assess phosphosignalling state. Uncropped images of the western blots are shown in Supplementary Fig. 9.

Immunoprecipitation. 293Ts stably expressing full-length PDFGR $\beta$-GFP or FGFR1-GFP were seeded in 10 -cm plates. When $\sim 70 \%$ confluent, each plate was transfected with $1 \mu \mathrm{g} 6 \mathrm{xHis}-\mathrm{SH} 2$-mCh-Cry2 plasmid DNA diluted in $9 \mu \mathrm{g}$ pBluescript by the calcium phosphate method. Twenty-four hours after transfection, cells were serum-starved overnight. Light-treated plates were illuminated with a 19-LED array for $10 \mathrm{~min}$ at $25^{\circ} \mathrm{C}$, after which cells were lysed in $1 \mathrm{ml}$ RIPA buffer. After incubation on ice for $30 \mathrm{~min}$, the cell lysate was spun at $4^{\circ} \mathrm{C}$ for $10 \mathrm{~min}$, and the supernatant from each sample was incubated with $5 \mu \mathrm{g}$ of anti-GFP antibody (Life Technologies, A-11122). Antibody-lysate solutions were incubated overnight at $4{ }^{\circ} \mathrm{C}$ with gentle shaking and were then incubated in $12.5 \mu \mathrm{l}$ immobilized protein A/G agarose beads (Thermo Scientific no. 20421) for $1 \mathrm{~h}$ as per the manufacturer's instructions. Beads were spun down and washed with lysis buffer three times, followed by one final wash with water. Beads were then incubated in $2 \times$ SDS sample buffer, boiled for $10 \mathrm{~min}$, spun down one final time, separated via SDS-PAGE and blotted on a nitrocellulose membrane. Blots were then probed for GFP or mCherry (Clontech no. 632543, 1:1,000 dilution).

Statistical methodology. Statistical power in luciferase assays was determined through the nonparametric Mann-Whitney Wilcoxon test. For comparing lamellipodial extension delays, varying dosages for the RTKi and PDGFRi treatment were compared using one-way ANOVA followed by Tukey post hoc pairwise analysis, with a significance threshold adjusted using the Bonferroni correction. FGFRi inhibition was compared with untreated cells using a Student's $t$-test. All statistical analyses were performed using the JMP statistical software suite.

\section{References}

1. Coward, P. et al. Controlling signaling with a specifically designed Gi-coupled receptor. Proc. Natl Acad. Sci. USA 95, 352-357 (1998)

2. Spencer, D., Wandless, T., Schreiber, S. \& Crabtree, G. Controlling signal transduction with synthetic ligands. Science 262, 1019-1024 (1993).

3. Dikic, I., Schlessinger, J. \& Lax, I. PC12 cells overexpressing the insulin receptor undergo insulin-dependent neuronal differentiation. Curr. Biol. 4, 702-708 (1994).

4. Jiang, Y., Woronicz, J. D., Liu, W. \& Goeddel, D. V. Prevention of constitutive TNF receptor 1 signaling by silencer of death domains. Science 283, 543-546 (1999).

5. Traverse, S. et al. EGF triggers neuronal differentiation of PC12 cells that overexpress the EGF receptor. Curr. Biol. 4, 694-701 (1994).

6. Snuderl, M. et al. Mosaic amplification of multiple receptor tyrosine kinase genes in glioblastoma. Cancer Cell 20, 810-817 (2011).

7. Prelich, G. Gene overexpression: uses, mechanisms, and interpretation. Genetics 190, 841-854 (2012).

8. Fenno, L., Yizhar, O. \& Deisseroth, K. The development and application of optogenetics. Annu. Rev. Neurosci. 34, 389-412 (2011).

9. Airan, R. D., Thompson, K. R., Fenno, L. E., Bernstein, H. \& Deisseroth, K. Temporally precise in vivo control of intracellular signalling. Nature 458, 1025-1029 (2009).

10. Wu, Y. I. et al. A genetically encoded photoactivatable Rac controls the motility of living cells. Nature 461, 104-108 (2009).

11. Levskaya, A., Weiner, O. D., Lim, W. A. \& Voigt, C. A. Spatiotemporal control of cell signalling using a light-switchable protein interaction. Nature 461, 997-1001 (2009).

12. Yazawa, M., Sadaghiani, A. M., Hsueh, B. \& Dolmetsch, R. E. Induction of protein-protein interactions in live cells using light. Nat. Biotechnol. 27, 941-945 (2009).

13. Kennedy, M. J. et al. Rapid blue-light-mediated induction of protein interactions in living cells. Nat. Method 7, 973-975 (2010).

14. Strickland, D. et al. TULIPs: tunable, light-controlled interacting protein tags for cell biology. Nat. Method 9, 379-384 (2012).

15. Zhou, X. X., Chung, H. K., Lam, A. J. \& Lin, M. Z. Optical control of protein activity by fluorescent protein domains. Science 338, 810-814 (2012).

16. Bugaj, L. J., Choksi, A. T., Mesuda, C. K., Kane, R. S. \& Schaffer, D. V. Optogenetic protein clustering and signaling activation in mammalian cells. Nat. Methods 10, 249-252 (2013).

17. Cao, J. et al. Light-inducible activation of target mRNA translation in mammalian cells. Chem. Commun. 49, 8338-8340 (2013).

18. Davis, S. et al. Ligands for EPH-related receptor tyrosine kinases that require membrane attachment or clustering for activity. Science 266, 816-819 (1994). 
19. Heldin, C.-H. Dimerization of cell surface receptors in signal transduction. Cell 80, 213-223 (1995).

20. Letourneur, F. \& Klausner, R. D. T-cell and basophil activation through the cytoplasmic tail of T-cell-receptor zeta family proteins. Proc. Natl Acad. Sci. USA 88, 8905-8909 (1991).

21. Margadant, C., Monsuur, H. N., Norman, J. C. \& Sonnenberg, A. Mechanisms of integrin activation and trafficking. Curr. Opin. Cell Biol. 23, 607-614 (2011).

22. Romeo, C. \& Seed, B. Cellular immunity to HIV activated by CD4 fused to T cell or Fc receptor polypeptides. Cell 64, 1037-1046 (1991).

23. Schlessinger, J. Cell signaling by receptor tyrosine kinases. Cell 103, 211-225 (2000).

24. Zhao, Y., Tong, C. \& Jiang, J. Hedgehog regulates smoothened activity by inducing a conformational switch. Nature 450, 252-258 (2007).

25. Cong, F., Schweizer, L. \& Varmus, H. Wnt signals across the plasma membrane to activate the $\beta$-catenin pathway by forming oligomers containing its receptors, Frizzled and LRP. Development 131, 5103-5115 (2004).

26. Duchemin, A. M., Ernst, L. K. \& Anderson, C. L. Clustering of the high affinity Fc receptor for immunoglobulin $\mathrm{G}$ (Fc gamma RI) results in phosphorylation of its associated gamma-chain. J. Biol. Chem. 269, 12111-12117 (1994).

27. Havranek, J. J. \& Harbury, P. B. Automated design of specificity in molecular recognition. Nat. Struct. Mol. Biol. 10, 45-52 (2003).

28. Metcalfe, C., Mendoza-Topaz, C., Mieszczanek, J. \& Bienz, M. Stability elements in the LRP6 cytoplasmic tail confer efficient signalling upon DIXdependent polymerization. J. Cell Sci. 123, 1588-1599 (2010).

29. Fuerer, C. \& Nusse, R. Lentiviral vectors to probe and manipulate the Wnt signaling pathway. PLoS ONE 5, e9370 (2010).

30. Zippel, R. et al. Inhibition of phosphotyrosine phosphatases reveals candidate substrates of the PDGF receptor kinase. Eur. J. Cell Biol. 50, 428-434 (1989).

31. Mohammadi, M. et al. A tyrosine-phosphorylated carboxy-terminal peptide of the fibroblast growth factor receptor (Flg) is a binding site for the SH2 domain of phospholipase C-gamma 1. Mol. Cell Biol. 11, 5068-5078 (1991).

32. Anderson, D. et al. Binding of SH2 domains of phospholipase C gamma 1, GAP, and Src to activated growth factor receptors. Science 250, 979-982 (1990).

33. Sieg, D. J. et al. FAK integrates growth-factor and integrin signals to promote cell migration. Nat. Cell Biol. 2, 249-256 (2000).

34. Rosenfeldt, H. M. et al. EDG-1 links the PDGF receptor to Src and focal adhesion kinase activation leading to lamellipodia formation and cell migration. FASEB J. 15, 2649-2659 (2001)

35. Kundra, V. et al. Regulation of chemotaxis by the platelet-derived growth factor receptor-[beta]. Nature 367, 474-476 (1994).

36. Heldin, C.-H. \& Westermark, B. Mechanism of action and in vivo role of platelet-derived growth factor. Physiol. Rev. 79, 1283-1316 (1999).

37. Watton, S. J. \& Downward, J. Akt/PKB localisation and $3^{\prime}$ phosphoinositide generation at sites of epithelial cell-matrix and cell-cell interaction. Curr. Biol. 9, 433-436 (1999)

38. Maheshwari, G., Brown, G., Lauffenburger, D. A., Wells, A. \& Griffith, L. G. Cell adhesion and motility depend on nanoscale RGD clustering. J. Cell Sci. 113, 1677-1686 (2000).

39. Li, R. et al. Activation of integrin $\alpha \mathrm{IIb} \beta 3$ by modulation of transmembrane helix associations. Science 300, 795-798 (2003).

40. Buensuceso, C., de Virgilio, M. \& Shattil, S. J. Detection of integrin $\alpha$ IIb $\beta 3$ clustering in living cells. J. Biol. Chem. 278, 15217-15224 (2003).

41. Anthis, N. J. et al. $\beta$ integrin tyrosine phosphorylation is a conserved mechanism for regulating talin-induced integrin activation. J. Biol. Chem. 284, 36700-36710 (2009).
42. Bouvard, D., Pouwels, J., De Franceschi, N. \& Ivaska, J. Integrin inactivators: balancing cellular functions in vitro and in vivo. Nat. Rev. Mol. Cell Biol. 14, 430-442 (2013).

43. Kraynov, V. S. et al. Localized Rac activation dynamics visualized in living cells Science 290, 333-337 (2000).

44. Habib, S. J. et al. A localized Wnt signal orients asymmetric stem cell division in vitro. Science 339, 1445-1448 (2013).

45. Purvis, J. E. et al. p53 dynamics control cell fate. Science 336, 1440-1444 (2012)

46. Wang, X., Chen, X. \& Yang, Y. Spatiotemporal control of gene expression by a light-switchable transgene system. Nat. Method 9, 266-269 (2012).

47. Chang, K.-Y. et al. Light-inducible receptor tyrosine kinases that regulate neurotrophin signalling. Nat. Commun. 5, 4057 (2014).

48. Kim, N. et al. Spatiotemporal control of fibroblast growth factor receptor signals by blue light. Chem. Biol. 21, 903-912 (2014).

49. Hyland, S., Beerli, R. R., Barbas, III C. F., Hynes, N. E. \& Wels, W. Generation and functional characterization of intracellular antibodies interacting with the kinase domain of human EGF receptor. Oncogene 22, 1557-1567 (2003).

50. Ozkan-Dagliyan, I. et al. Formation of Arabidopsis Cryptochrome 2 photobodies in mammalian nuclei: application as an optogenetic DNA damage checkpoint switch. J. Biol. Chem. 288, 23244-23251 (2013).

51. Taslimi, A. et al. An optimized optogenetic clustering tool for probing protein interaction and function. Nat. Commun. 5, 4925 (2014).

52. Quan, J. \& Tian, J. Circular polymerase extension cloning of complex gene libraries and pathways. PLoS ONE 4, e6441 (2009).

53. Gage, F. H. et al. Survival and differentiation of adult neuronal progenitor cells transplanted to the adult brain. Proc. Natl Acad. Sci. USA 92, 11879-11883 (1995).

54. Yu, J. H. \& Schaffer, D. V. Selection of novel vesicular stomatitis virus glycoprotein variants from a peptide insertion library for enhanced purification of retroviral and lentiviral vectors. J. Virol. 80, 3285-3292 (2006).

\section{Acknowledgements}

We thank Dr M. Niewiadomska-Bugaj (Western Michigan University) for assistance with statistical analysis, and E. Connelly, N. Repina, A. Choksi and S. Pandit for experimental assistance. This work was supported by the Department of Energy Award DE-SC0001216, NIH R01 NS087253, and funding from the Siebel Stem Cell Institute (to D.V.S.) and Siebel Scholars Foundation (L.J.B. and D.P.S.)

\section{Author contributions}

L.J.B. conceived and directed the study, designed and performed experiments, and analysed data. D.P.S., C.K.M. and M.V. provided experimental assistance and performed experiments. D.V.S. provided conceptual and technical guidance and support. L.J.B. wrote the manuscript, with revision and editing from D.V.S. and R.S.K.

\section{Additional information}

Supplementary Information accompanies this paper at http://www.nature.com/ naturecommunications

Competing financial interests: The authors declare no competing financial interests

Reprints and permission information is available online at http://npg.nature.com/ reprintsandpermissions/

How to cite this article: Bugaj, L. J. et al. Regulation of endogenous transmembrane receptors through optogenetic Cry2 clustering. Nat. Commun. 6:6898 doi: $10.1038 /$ ncomms7898 (2015). 